\title{
Association between copeptin levels and treatment responses to hypertonic saline infusion in patients with symptomatic hyponatremia: a prospective cohort study
}

\author{
Suryeong $\mathrm{Go}^{1}$, Sejoong $\mathrm{Kim}^{2}$, Hyung-Eun Son ${ }^{2}$, Ji-Young Ryu ${ }^{2}$, Huijin Yang ${ }^{3}$, Sun Ryoung Choi ${ }^{3}$, Jang-Won Seo ${ }^{3}$, \\ You Hwan Jo ${ }^{4}$, Ja-Ryong Koo ${ }^{3}$, Seon Ha Baek ${ }^{3}$
}

${ }^{1}$ Department of Internal Medicine, Armed Forces Yangju Hospital, Yangju, Republic of Korea

2 Department of Internal Medicine, Seoul National University Bundang Hospital, Seongnam, Republic of Korea

${ }^{3}$ Department of Internal Medicine, Hallym University Dongtan Sacred Heart Hospital, Hwaseong, Republic of Korea

${ }^{4}$ Department of Emergency Medicine, Seoul National University Bundang Hospital, Seongnam, Republic of Korea

Background: Copeptin is secreted in equimolar amounts as arginine vasopressin, main hormone regulating body fluid homeostasis. A recent study reported a copeptin-based classification of osmoregulatory defects in syndromes of inappropriate antidiuresis that may aid in prediction of therapeutic success. We investigated usefulness of copeptin for differentiating etiologies of hyponatremia and predicting efficacy and safety of hypertonic saline treatment in hyponatremic patients.

Methods: We performed a multicenter, prospective cohort study of 100 inpatients with symptomatic hyponatremia (corrected serum sodium [s $\mathrm{sa}$ ] $\leq 125 \mathrm{mmol} / \mathrm{L}$ ) treated with hypertonic saline. Copeptin levels were measured at baseline and 24 hours after treatment initiation, and patients were classified as being below or above median of copeptin at baseline or at 24 hours, respectively. Correlations between target, under correction, and overcorrection rates of sNa within 24 hours/24-48 hours and copeptin levels at baseline/24 hours were analyzed.

Results: Mean sNa and median copeptin levels were 117.9 and $16.9 \mathrm{pmol} / \mathrm{L}$, respectively. Ratio of copeptin-to-urine sodium allowed for an improved differentiation among some (insufficient effective circulatory volume), but not all hyponatremia etiologic subgroups. Patients with below-median copeptin levels at baseline achieved a higher target correction rate in 6/24 hours (odds ratio [OR], 2.97; $p=0.02 / O R, 6.21 ; p=0.006$ ). Patients with below-median copeptin levels 24 hours after treatment showed a higher overcorrection rate in next 24 hours $(\mathrm{OR}, 18.00, \mathrm{p}=0.02)$.

Conclusion: There is a limited diagnostic utility of copeptin for differential diagnosis of hyponatremia. However, copeptin might be useful for predicting responses to hypertonic saline treatment in hyponatremic patients.

Keywords: Copeptins, Diagnosis, Hypertonic saline solution, Hyponatremia, Treatment outcome

Received: November 23, 2020; Revised: April 20, 2021; Accepted: April 28, 2021

Editor: Soo Wan Kim, Chonnam National University, Gwangju, Republic of Korea

Correspondence: Seon Ha Baek Department of Internal Medicine, Hallym University Dongtan Sacred Heart Hospital, 7 Keunjaebong-gil,

Hwaseong 18450, Republic of Korea.E-mail: seonhabaek@hallym.or.kr, haya2001@hanmail.net

ORCID: https://orcid.org/0000-0002-4751-9817

Suryeong Go and Sejoong Kim contributed equally to this work as co-first authors.

Copyright (C) 2021 by The Korean Society of Nephrology

(a) This is an Open Access article distributed under the terms of the Creative Commons Attribution Non-Commercial and No Derivatives License (http:// creativecommons.org/licenses/by-nc-nd/4.0/) which permits unrestricted non-commercial use, distribution of the material without any modifications, and reproduction in any medium, provided the original works properly cited. 


\section{Introduction}

Hyponatremia is the most common electrolyte abnormality encountered in clinical practice. It is associated with increased mortality and morbidity rates that arise from the abnormality itself and from treatment errors [1,2]. Hypertonic saline infusion is generally accepted as the treatment of choice for severe symptomatic hyponatremia [2,3]. However, overcorrection of chronic hyponatremia may lead to osmotic demyelination syndrome (ODS), causing permanent neurological disability, while an under correction may be insufficient to prevent the life-threatening manifestations of cerebral edema [4-6].

In most cases, hyponatremia occurs due to the inappropriate activation of arginine vasopressin (AVP) $[7,8]$. Therefore, AVP can be used for the diagnosis and prediction of treatment response [9]. However, due to its short half-life (24 minutes) and high platelet affinity, it is difficult to precisely measure AVP levels. Therefore, AVP levels are not commonly used in clinical practice for hyponatremia diagnosis [10]. Copeptin has recently been reported to be highly correlated with AVP in response to hypoosmolar or stressful conditions [11]. Copeptin is a stable, nonfunctional, 39-amino acid glycopeptide. It forms the C-terminal of pre-proAVP, which is a precursor peptide composed of a signal peptide, AVP, and neurophysin II [12]. Unlike AVP, copeptin is highly stable in terms of storage at room temperature and can be easily detected by sandwich immunoassay. Furthermore, its level correlates well with AVP levels $[11,13]$. Therefore, copeptin is a promising alternative marker of AVP.

Several studies have investigated the role of copeptin in a variety of conditions, including its role in: (1) the diagnosis of central diabetes insipidus caused by a primary AVP-synthesis defect $[14,15]$; (2) early detection and exclusion of acute myocardial infarction [16,17]; and (3) the prognosis of heart failure, sepsis, chronic renal failure, liver cirrhosis, and polycystic kidney disease [18-21]. Furthermore, in terms of pathophysiology, several reports have shown its capability to differentiate the underlying cause of hyponatremia, using copeptin/urine $\mathrm{Na}(\mathrm{UNa})$ ratios or copeptin itself to distinguish primary AVP release (syndrome of inappropriate antidiuresis [SIAD]) and AVP release secondary to hemodynamic stimuli, including heart failure or Na depletion [10,22-24]. However, copeptin levels widely overlap in patients with hyponatremia [9] and vary among patients with SIAD [8]. Physiological increases in copeptin following hypertonic saline-induced osmotic stimulation have been observed in a healthy population [8]. However, the response of copeptin to hypertonic saline infusion varied substantially in patients with SIAD according to impaired osmoregulation or nonosmotic inhibitory pathway [8]. Defining SIAD subtypes according to copeptin responses to osmotic stimulation by hypertonic saline infusion may serve as the starting point for assessing individual treatment responses in SIAD [8]. However, only a few studies have used copeptin to predict responses to treatment with hypertonic saline in hyponatremic patients using clinical indices such as target, under correction, and overcorrection rates. Therefore, this study aimed to evaluate copeptin levels as a marker for responses to hypertonic saline treatment in hyponatremic patients.

\section{Methods}

\section{Study population}

A multicenter, prospective, randomized clinical trial was designed to evaluate the efficacy and safety of rapid intermittent correction compared with slow continuous correction with hypertonic saline in patients with symptomatic hyponatremia (SALSA trial) (Clinicaltrials.gov: NCT02887469) [25]. The detailed study protocol is described elsewhere [26]. In brief, the study participants with symptomatic hyponatremia were recruited from August 24, 2016 to August 21, 2019. Inclusion criteria for the original study included: age $>18$ years and symptomatic hyponatremia (glucose-corrected serum $\mathrm{Na}[\mathrm{sNa}] \leq 125 \mathrm{mmol} / \mathrm{L}$ ). Table 1 summarizes the inclusion and exclusion criteria used in the SALSA study.

Of the 178 participants from the SALSA study, 62 (34.8\%) who had available data for copeptin at baseline and 24 hours after initiation of hypertonic saline treatment were included in the present analyses. We further prospectively recruited 38 patients with symptomatic hyponatremia who were treated with hypertonic saline between May 2018 and July 2019, with the same inclusion and exclusion criteria as the SALSA study (Fig. 1). Finally, using a total of 100 patients with symptomatic hyponatremia who were treated with hypertonic saline, the usefulness of copeptin as a diagnostic and responsive marker for hypertonic saline was evaluated at the Hallym University Dongtan Sacred Heart Hospital and the Seoul National University Bundang Hospital. All clinical 
Table 1. Inclusion and exclusion criteria of SALSA study

\begin{tabular}{|c|c|}
\hline Inclusion criteria & Exclusion criteria \\
\hline - Age over 18 years & - Primary polydipsia (urine osmolality $\leq 100 \mathrm{mOsm} / \mathrm{kg}$ ) \\
\hline - Glucose-corrected serum Na $\leq 125 \mathrm{mmol} / \mathrm{L}$ & $\begin{array}{l}\text { - Arterial hypotension (systolic blood pressure }<90 \mathrm{mmHg} \text { and mean arterial pressure }< \\
70 \mathrm{mmHg} \text { ) }\end{array}$ \\
\hline \multirow[t]{4}{*}{ - Symptoms } & $\begin{array}{l}\text { - Liver disease (transaminase levels }>3 \text { times the upper limit of normal, known decom- } \\
\text { pensated liver cirrhosis with ascites or diuretic use, hepatic encephalopathy, and } \\
\text { varices) }\end{array}$ \\
\hline & - Uncontrolled diabetes mellitus (glycated hemoglobin> 9\%) \\
\hline & $\begin{array}{l}\text { - Had a history of cardiac surgery, acute myocardial infarction, sustained ventricular tachy- } \\
\text { cardia, ventricular fibrillation, acute coronary syndrome, cerebral trauma, and increased } \\
\text { intracranial pressure within } 3 \text { months prior to randomization }\end{array}$ \\
\hline & - Pregnant or breast feeding \\
\hline
\end{tabular}

SALSA, a randomized clinical trial to evaluate the efficacy and safety of rapid intermittent correction compared with slow continuous correction with hypertonic saline in patients with symptomatic hyponatremia.

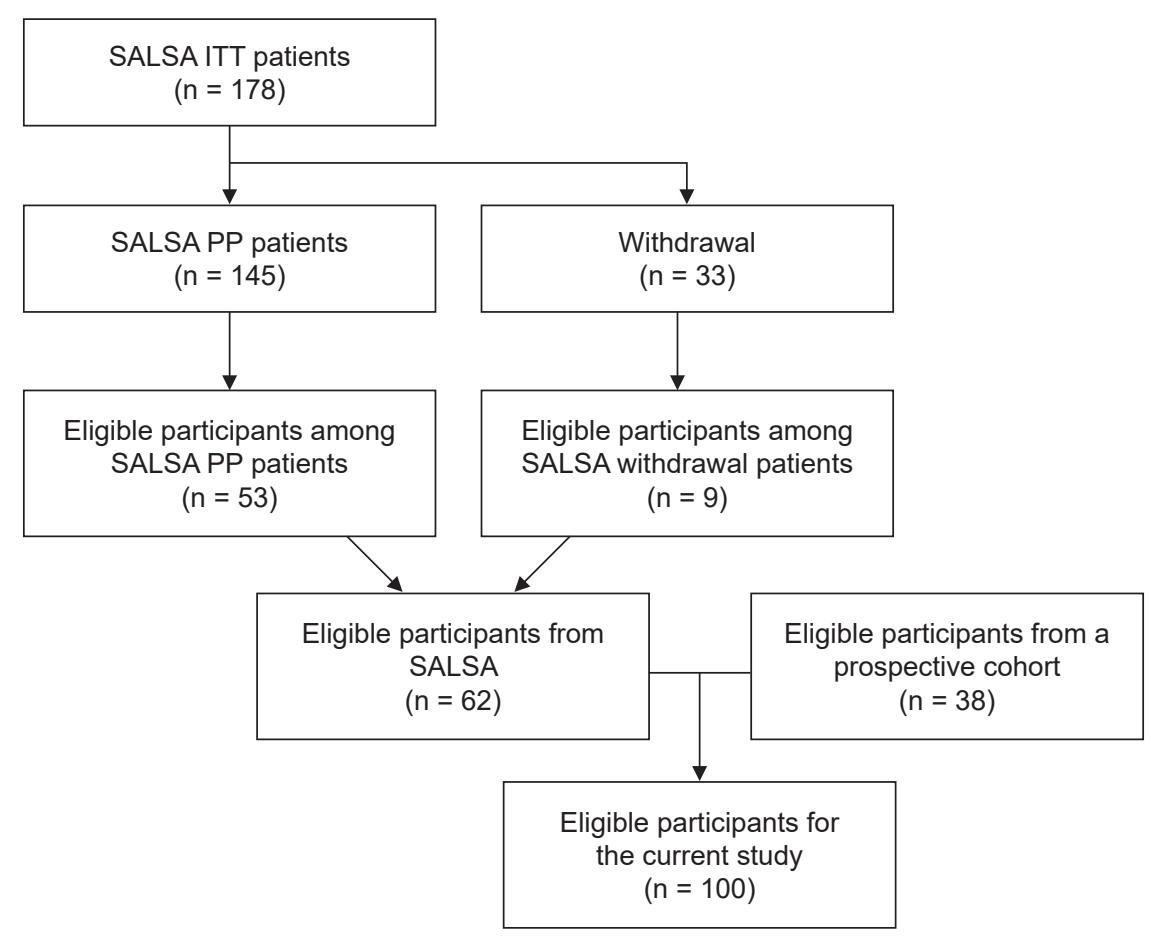

Figure 1. Study population algorithm.

ITT, intention to treat; PP, per protocol; SALSA, a randomized clinical trial to evaluate the efficacy and safety of rapid intermittent correction compared with slow continuous correction with hypertonic saline in patients with symptomatic hyponatremia.

investigations were conducted in accordance with the 2008 Declaration of Helsinki principles and good clinical practice guidelines. This study was approved by the Institutional Review Boards of the Hallym University Dongtan Sacred Heart Hospital (No. 2018-04-009) and the Seoul National University Bundang Hospital (No. H-1508-310-115). Written informed consent was obtained from all patients.

\section{Data collection and definitions}

Anthropometric markers (including height and weight) and resting systolic and diastolic blood pressure were measured at baseline. Based on the International Classification of Disease (10th revision) codes, comorbidities such as hypertension, diabetes mellitus, congestive heart failure, adrenal insufficiency, hypothyroidism, and cancer were assessed 
by screening for the I10-I15; E10-14; I11.0, I13.0, I13.2, I50; E27.1-27.4; E03; and C codes, respectively. The presence of all comorbidities was determined by a self-reported history or medical record review, and the presence of hypertension, diabetes mellitus, and hypothyroidism was confirmed on the basis of a history of antihypertensive medications, antihyperglycemic agents, and levothyroxine use, respectively. Hypothyroidism was defined as a thyroid-stimulating hormone concentration above the reference range (0.4-4.0 mIU/L) and free thyroxine concentration below the reference range. Adrenal insufficiency was defined as a basal cortisol level below $3 \mu \mathrm{g} / \mathrm{dL}$ or plasma cortisol level below $18 \mu \mathrm{g} / \mathrm{dL} 30$ to 60 minutes after $250-\mu \mathrm{g}$ cosyntropin administration. The amounts of hypertonic saline administered were recorded for 48 hours. Concentrations of sNa were measured via an indirect ion-selective electrode method using the AU5800 (Beckman Coulter, Brea, CA, USA) at the Hallym University Dongtan Sacred Heart Hospital and Seoul National University Bundang Hospital, and the Dimension Vista 1500 (Siemens Healthineers, Erlangen, Germany) at Seoul National University Bundang Hospital.

\section{Biochemistry measurement and analysis}

Blood samples for the determination of copeptin were collected at baseline and 24 to 48 hours after the initiation of hypertonic saline. After centrifugation at 2,000 $\times g$, the samples were frozen at $-70^{\circ} \mathrm{C}$ until assayed. Serum copeptin concentrations were determined in a single batch using a commercial automated immunofluorescence assay (B.R.A.H.M.S KRYPTOR Copeptin proAVP; Thermo Scientific Biomarkers, Hennigsdorf, Germany).

\section{Study outcomes}

To determine the underlying cause of hyponatremia, a structured diagnostic approach was implemented [1]. This approach was based on the patient's history, physical examination, and laboratory test results. All patients were classified into five categories: (1) decreased extracellular fluid (ECF) volume due to renal Na loss (e.g., diuretics, especially thiazides); (2) decreased ECF volume due to non-renal Na loss (e.g., gastrointestinal Na loss or third spacing: vomiting, diarrhea, or malnutrition); (3) increased ECF volume (e.g., heart failure, liver cirrhosis, and nephrotic syndrome); (4) normal
ECF volume with adrenal insufficiency; and (5) normal ECF volume fulfilling the essential criteria for $\operatorname{SIAD}[1,27]$

The overcorrection rate, a surrogate marker of ODS, was defined as follows: an increase in the sNa level above 12 $\mathrm{mmol} / \mathrm{L}$ within the first 24 hours or above $18 \mathrm{mmol} / \mathrm{L}$ within the first 24 to 48 hours based on previously published criteria [28] and recent guidelines [1,7]. Target correction rates were defined as sNa levels of 5 to $9 \mathrm{mmol} / \mathrm{L}$ within 24 hours and 10 to $17 \mathrm{mmol} / \mathrm{L}$ within 24 to $48 \mathrm{hours}$ or $\geq 130 \mathrm{mmol} / \mathrm{L}$. Under correction rates were defined as sNa levels of less than 5 $\mathrm{mmol} / \mathrm{L}$ within 24 hours and less than $10 \mathrm{mmol} / \mathrm{L}$ within 24 to 48 hours.

\section{Hypertonic saline infusion protocol}

Participants from the SALSA trial underwent either rapid intermittent correction or slow continuous correction with hypertonic saline after randomization, as previously reported [26]. Participants from the prospective cohort underwent either rapid intermittent correction and/or slow continuous correction with no formalized protocol, according to the physician's discretion.

\section{Statistical analysis}

The baseline characteristics and laboratory data are expressed as means \pm standard deviations for normally distributed variables, medians (interquartile range) for non-normally distributed variables, and frequencies and percentages for categorical variables. The chi-square and Fisher exact tests were used to analyze categorical variables. The intergroup differences in the continuous variables were analyzed using the analysis of variance, Kruskal-Wallis tests, and Bonferroni post hoc tests to account for multiple testing. To compare two disorders among the five groups, a Mann-Whitney test with Bonferroni post hoc analysis was used; $\mathrm{P}<0.005$ was considered statistically significant. To present the diagnostic utility of copeptin, we used the area under the receiver operator characteristic curve (AUROC). Logistic regression analysis was performed to evaluate the risk of under correction, target correction, and overcorrection. The odds ratios (ORs) and 95\% confidence intervals (CIs) for the risk of outcomes were calculated after stepwise adjustment for multiple confounders. Statistical significance was set at $\mathrm{p}<0.05$. All analyses were performed using IBM 
SPSS version 26.0 (IBM Corp., Armonk, NY, USA) and STATA (version 14.0; StataCorp LP, College Station, TX, USA).

\section{Results}

\section{Study population}

Table 2 shows baseline patient characteristics and biochemical data. The mean age and sNa level were 73 years and $117.9 \mathrm{mmol} / \mathrm{L}$, respectively. The causes of hyponatremia were renal loss due to thiazide use, decreased ECF due to non-renal Na loss, increased ECF, adrenal insufficiency only, and SIAD in $30 \%, 9 \%, 11 \%, 11 \%$, and $39 \%$ of the patients, respectively. The infusion modes of hypertonic saline were bolus therapy, continuous infusion, and mixed type in $45 \%$, $54 \%$, and $1 \%$ of the patients, respectively.

\section{Copeptin levels and the copeptin-to-urine $\mathrm{Na}$ ratio ac- cording to the cause of hyponatremia}

The overall median copeptin level and copeptin-to-UNa ratio $(\times 100)$ at baseline were $16.9 \mathrm{pmol} / \mathrm{L}(7.6-43.8 \mathrm{pmol} /$ L) and $28.0 \mathrm{pmol} / \mathrm{mol}(11.6-88.3 \mathrm{pmol} / \mathrm{mol})$, respectively. The median copeptin and copeptin-to-UNa ratios are expressed as standard box plots according to the cause of hyponatremia (Fig. 2). Although there were no differences in the copeptin levels among the five groups, the copeptinto-UNa ratio differed significantly according to the cause of hyponatremia $(p=0.001)$. The copeptin-to-UNa ratios in disorders with low effective arterial blood volume, secondary copeptin secretion (increased ECF or decreased ECF secondary to non-renal $\mathrm{Na}$ loss) tended to be higher than those with other causes of hyponatremia. The copeptinto-UNa ratio exhibited a diagnostic utility identifying low effective arterial volume with an AUROC value of 0.78 (95\% CI, 0.65-0.91, p < 0.001) (Fig. 3). The copeptin-to-UNa ratio in the group with decreased ECF due to non-renal Na loss was significantly higher than that in the groups of thiazide use ( $\mathrm{p}<0.001)$, adrenal insufficiency $(\mathrm{p}<0.001)$, and SIAD ( $\mathrm{p}$ $<0.001)$; however, there were no differences in the copeptin levels or the copeptin-to-UNa ratios among the three groups. Furthermore, there were no differences in copeptin levels or copeptin-to-UNa ratios between groups with decreased ECF due to non-renal Na loss and increased ECF (Table 3; Supplementary Table 1, available online).
Table 2. Baseline characteristics at initiation of study

\begin{tabular}{|c|c|}
\hline Characteristic & Total $(n=100)$ \\
\hline \multicolumn{2}{|l|}{ Demographic } \\
\hline Male sex & $48(48.0)$ \\
\hline Age (yr) & $73.0 \pm 12.1$ \\
\hline Weight (kg) & $57.8 \pm 10.9$ \\
\hline Body mass index $\left(\mathrm{kg} / \mathrm{m}^{2}\right)$ & $22.9 \pm 4.9$ \\
\hline \multicolumn{2}{|l|}{ Comorbidity } \\
\hline Diabetes mellitus & $29(29.0)$ \\
\hline Hypertension & $64(64.0)$ \\
\hline Congestive heart failure & $6(6.0)$ \\
\hline Liver cirrhosis & $2(2.0)$ \\
\hline Nephrotic syndrome & $6(6.0)$ \\
\hline Hypothyroidism & $6(6.0)$ \\
\hline Malignancy & $23(23.0)$ \\
\hline \multicolumn{2}{|l|}{ Hyponatremia cause } \\
\hline Thiazide use & $30(30.0)$ \\
\hline Decreased ECF due to non-renal sodium loss & $9(9.0)$ \\
\hline Increased ECF & $11(11.0)$ \\
\hline Adrenal insufficiency & $11(11.0)$ \\
\hline Syndrome of inappropriate antidiuresis & $39(39.0)$ \\
\hline $\begin{array}{l}\text { Infusion mode of hypertonic saline (bolus/contin- } \\
\text { uous/mixed) }\end{array}$ & $45 / 54 / 1$ \\
\hline Systolic blood pressure (mmHg) & $132.5 \pm 22.8$ \\
\hline Diastolic blood pressure (mmHg) & $74.4 \pm 14.2$ \\
\hline \multicolumn{2}{|l|}{ Laboratory value } \\
\hline $\mathrm{Na}(\mathrm{mmol} / \mathrm{L})$ & $117.9 \pm 5.3$ \\
\hline Serum osmolality (mOsm/kg) & $250.7 \pm 18.9$ \\
\hline Creatinine (mg/dL) & $1.04 \pm 1.06$ \\
\hline White blood cell $\left(10^{9} / \mathrm{L}\right)$ & $8.4 \pm 4.3$ \\
\hline Hemoglobin (g/dL) & $11.7 \pm 1.9$ \\
\hline Albumin (g/dL) & $4.0 \pm 0.6$ \\
\hline $\mathrm{Ca}(\mathrm{mg} / \mathrm{dL})$ & $8.7 \pm 0.7$ \\
\hline$P(\mathrm{mg} / \mathrm{dL})$ & $3.2 \pm 1.2$ \\
\hline $\mathrm{K}(\mathrm{mmol} / \mathrm{L})$ & $3.9 \pm 0.7$ \\
\hline Total $\mathrm{CO}_{2}(\mathrm{mmol} / \mathrm{L})$ & $23.3 \pm 5.7$ \\
\hline Uric acid (mg/dL) & $4.0 \pm 2.7$ \\
\hline Total cholesterol (mg/dL) & $155.0 \pm 50.4$ \\
\hline Total bilirubin (mg/dL) & $0.9 \pm 0.4$ \\
\hline AST (IU/L) & $31.1 \pm 17.3$ \\
\hline ALT (IU/L) & $17.2 \pm 8.9$ \\
\hline C-reactive protein (mg/L) & $25.8 \pm 34.4$ \\
\hline Urine osmolality (mOsm/kg) & $418.0 \pm 156.3$ \\
\hline Urine $\mathrm{Na}(\mathrm{mmol} / \mathrm{L})$ & $73.5 \pm 47.5$ \\
\hline Urine K (mmol/L) & $34.0 \pm 24.0$ \\
\hline
\end{tabular}

Data are expressed as number (\%), mean \pm standard deviation, or number only.

ALT, alanine aminotransferase; AST, aspartate aminotransferase; ECF, extracellular fluid. 

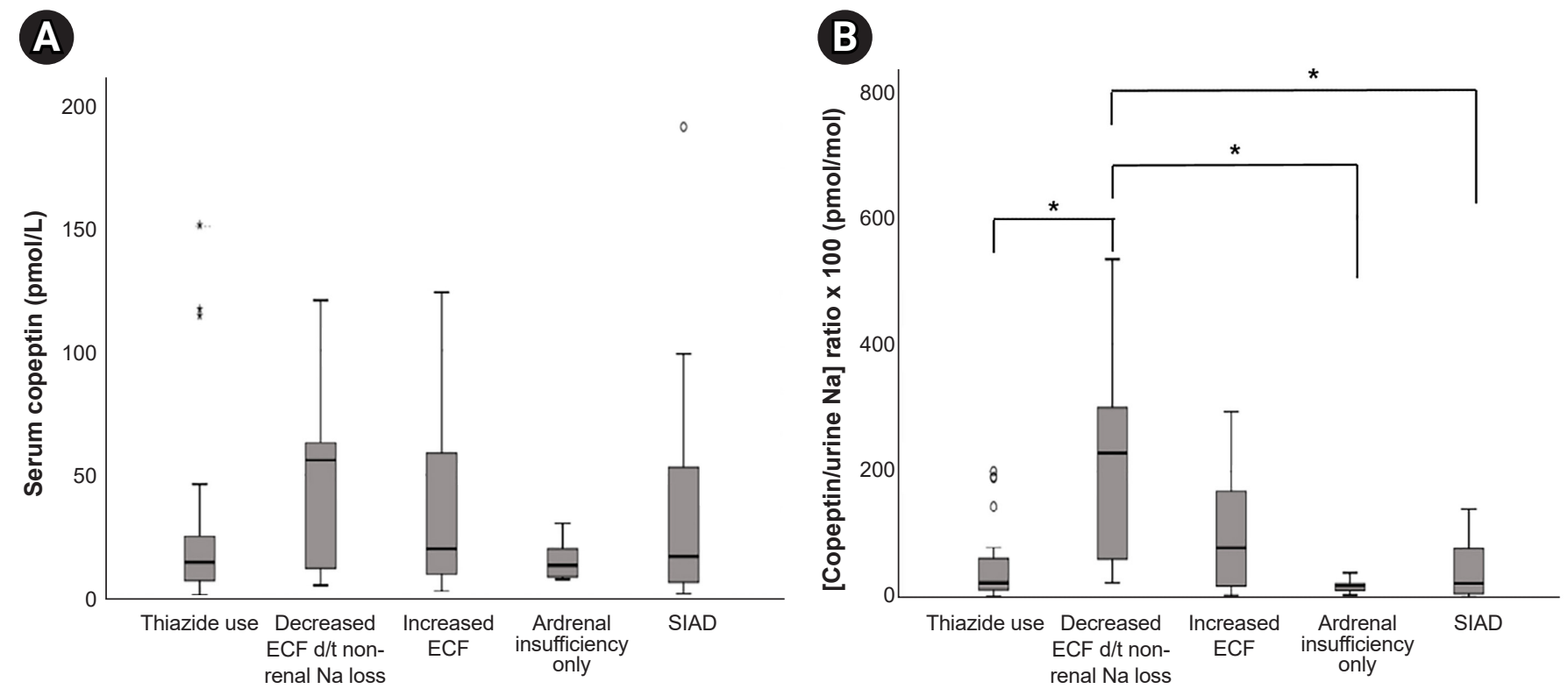

Figure 2. Box plot for copeptin levels $(A)$ and copeptin-to-urine $\mathrm{Na} \times \mathbf{1 0 0}(\mathrm{B})$ according to etiologies of hyponatremia.

ECF, extracellular fluid; SIAD, syndrome of inappropriate antidiuresis.

* $\mathrm{P}<0.005$, compared with decreased ECF due to non-renal Na loss (Kruskal-Wallis test and Bonferroni post hoc test).

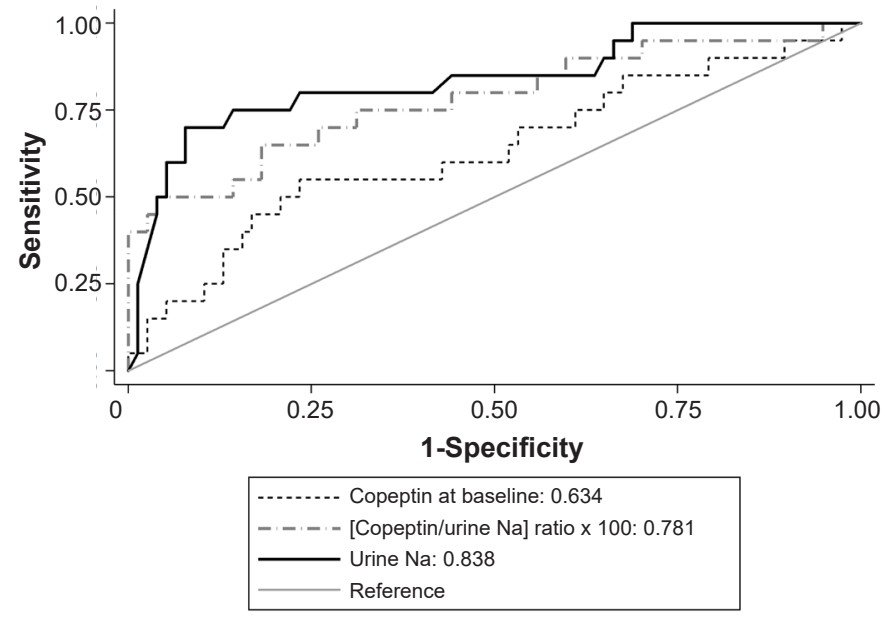

Figure 3. Area under receiver operating characteristics curve for insufficient effective arterial blood volume (secondary copeptin release).

Factors associated with serum copeptin levels at baseline

Table 4 shows the clinical and biochemical determinants of hyponatremic patients with copeptin levels above and below the median. Univariable analysis revealed that patients with copeptin levels above the median were characterized by lower sNa and serum potassium levels and a higher urine potassium level. Multivariable logistic regression analysis revealed that lower sNa levels (OR, 0.82; 95\% CI, 0.73-0.93) and higher serum creatinine (OR, 2.91, 95\% CI, 1.22-6.95) and urine potassium (OR, 1.05; 95\% CI, 1.02-1.09) levels were independently associated with copeptin levels above the median.

\section{Predicting responses to hypertonic saline}

Supplementary Table 2 (available online) shows the cumulative amounts of hypertonic saline by time frame according to the infusion methods. The cumulative amounts of hypertonic saline were $173.6 \mathrm{~mL}$ within 6 hours, $337.7 \mathrm{~mL}$ within 24 hours, and $521.4 \mathrm{~mL}$ within 48 hours.

Table 5, Fig. 4, and Fig. 5 show the frequency and adjusted ORs for associations between copeptin at baseline and 24 hours after hypertonic saline treatment and the outcomes. Under correction, target correction, and overcorrection within 6 hours after treatment occurred in 38, 47, and five patients, respectively. Evaluation of the relationship between copeptin levels at baseline and target correction rate using multivariable logistic regression, even after adjustment for covariates such as infusion methods (rapid intermittent correction or slow continuous correction) and amounts of 


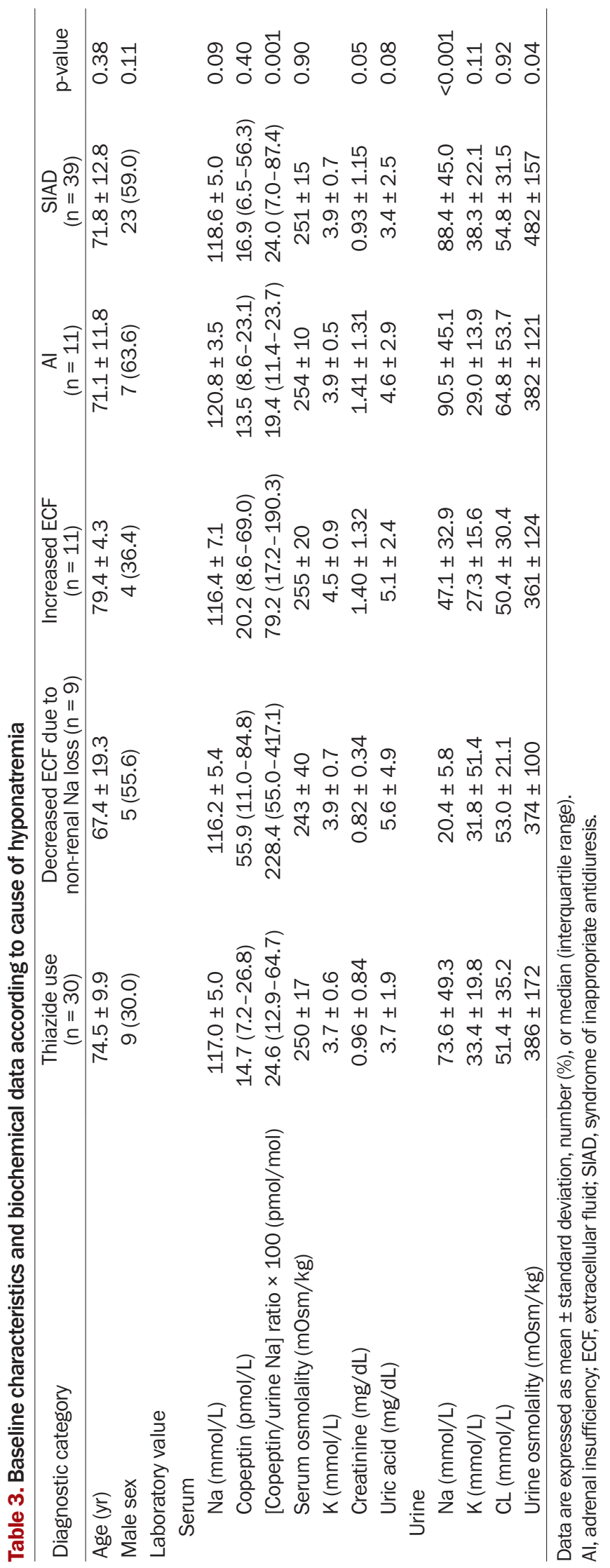

Table 4. Factors associated with serum copeptin (above/below the median) at baseline as a categorical variable in patients with hyponatremia

\begin{tabular}{lcc}
\hline Parameter & OR $(95 \% \mathrm{Cl})$ & p-value \\
\hline Univariable analysis & $1.00(0.97-1.04)$ & 0.88 \\
Age & $1.00(0.45-2.21)$ & $>0.99$ \\
Male sex & $0.84(0.36-1.92)$ & 0.67 \\
Hypertension & $1.50(0.62-3.62)$ & 0.37 \\
Diabetics vs. non-diabetics & $0.89(0.35-2.27)$ & 0.81 \\
Cancer & & 0.76 \\
Cause of hyponatremia & $1.02(0.94-1.11)$ & 0.68 \\
Body mass index & $0.99(0.97-1.01)$ & 0.27 \\
Systolic blood pressure & $0.85(0.78-0.94)$ & $0.001 *$ \\
Serum Na & $0.49(0.26-0.95)$ & $0.03 *$ \\
Serum K & $1.02(1.00-1.05)$ & 0.064 \\
Serum osmolality & $1.97(1.00-3.86)$ & 0.05 \\
Serum creatinine & $1.20(1.00-1.44)$ & 0.05 \\
Serum uric acid & $1.00(0.99-1.02)$ & 0.64 \\
Serum C-reactive protein & $1.00(1.00-1.00)$ & 0.74 \\
Urine osmolality & $0.99(0.99-1.00)$ & 0.16 \\
Urine Na & $1.04(1.01-1.06)$ & $0.002^{*}$ \\
Urine K & & \\
Multivariable analysis & & $0.002 *$ \\
Serum Na & $0.82(0.73-0.93)$ & $0.016 *$ \\
Serum creatinine & $2.91(1.22-6.95)$ & $0.002 *$ \\
Urine K & $1.05(1.02-1.09)$ & \\
\hline Cl, confidence interval; OR, odds ratio. & \\
*p < 0.05. & & \\
& &
\end{tabular}

hypertonic saline, revealed that baseline copeptin levels below the median had an increased probability of reaching target correction within 6 hours (OR, 2.97; 95\% CI, 1.16-7.64; $\mathrm{p}=0.02$ ). In terms of under correction and overcorrection within 6 hours, no differences were observed according to the copeptin levels (Fig. 4A). Under correction, target correction, and overcorrection occurred within 24 hours in 8 , 44 , and 22 patients, respectively. On multivariable logistic regression, even after adjustment for covariates including infusion methods and amounts of hypertonic saline, baseline copeptin levels below the median had an increased probability of reaching target correction within 24 hours (OR, 6.21; 95\% CI, 1.67-23.09; $\mathrm{p}=0.006$ ). In terms of under correction and overcorrection within 24 hours, no differences were observed based on the copeptin levels (Fig. 4B). Overall, the median copeptin levels at 24 hours and 48 hours after treatment were $7.9 \mathrm{pmol} / \mathrm{L}$ (4.7-20.6 pmol/L) and $7.5 \mathrm{pmol} / \mathrm{L}$ (4.3-18.4 pmol/L), respectively. Under correction, target correction, and overcorrection occurred within 24 to 48 hours in 28, 54, and 12 patients, respectively. Multivariable logistic 
Table 5. Adjusted ORs for association between copeptin below the median at baseline and outcomes

\begin{tabular}{|c|c|c|c|c|c|c|c|}
\hline \multirow{2}{*}{ Variable } & \multirow{2}{*}{$\begin{array}{l}\text { Number } \\
\text { (low, high) }\end{array}$} & \multicolumn{2}{|l|}{ Univariable } & \multicolumn{2}{|c|}{ Multivariablea } & \multicolumn{2}{|l|}{ Multivariableb } \\
\hline & & OR $(95 \% \mathrm{Cl})$ & p-value & OR $(95 \% \mathrm{Cl})$ & p-value & OR $(95 \% \mathrm{Cl})$ & $\mathrm{p}$-value \\
\hline \multicolumn{8}{|l|}{ Copeptin at baseline } \\
\hline Under correction within $6 \mathrm{hr}$ & $(17 / 49,21 / 49)$ & $0.71(0.31-1.60)$ & 0.41 & $0.70(0.31-1.60)$ & 0.40 & $0.32(0.10-1.02)$ & 0.05 \\
\hline Target correction within $6 \mathrm{hr}$ & $(30 / 49,17 / 49)$ & $2.97(1.31-6.76)$ & $0.009 *$ & $3.12(1.35-7.22)$ & $0.008 *$ & $2.97(1.16-7.64)$ & $0.02 *$ \\
\hline Overcorrection within $6 \mathrm{hr}$ & $(1 / 49,4 / 49)$ & $0.23(0.03-2.18)$ & 0.20 & $2.23(0.02-2.24)$ & 0.20 & $0.00(0.00)$ & $>0.99$ \\
\hline Under correction within $24 \mathrm{hr}$ & $(2 / 49,6 / 49)$ & $0.31(0.06-1.59)$ & 0.16 & $0.31(0.06-1.64)$ & 0.17 & $0.00(0.00)$ & 0.99 \\
\hline Target correction within $24 \mathrm{hr}$ & $(26 / 49,18 / 49)$ & $1.95(0.87-4.37)$ & 0.11 & $2.01(0.88-4.57)$ & 0.10 & $6.21(1.67-23.09)$ & $0.006 *$ \\
\hline Overcorrection within $24 \mathrm{hr}$ & $(11 / 49,11 / 49)$ & $1.00(0.39-2.58)$ & $>0.99$ & $0.92(0.34-2.51)$ & 0.87 & $0.44(0.07-2.77)$ & 0.379 \\
\hline \multicolumn{8}{|l|}{ Copeptin at $24 \mathrm{hr}$ after treatment } \\
\hline \multicolumn{2}{|c|}{ Under correction within $24-48 \mathrm{hr}(9 / 47,19 / 50)$} & $0.39(0.15-0.97)$ & $0.04 *$ & $0.38(0.15-0.97)$ & $0.04 *$ & $0.28(0.03-2.49)$ & 0.25 \\
\hline \multicolumn{2}{|c|}{ Target correction within $24-48 \mathrm{hr}(27 / 47,27 / 50)$} & $1.15(0.52-2.57)$ & 0.733 & $1.16(0.52-2.59)$ & 0.72 & $0.77(0.25-2.33)$ & 0.64 \\
\hline Overcorrection within $24-48 \mathrm{hr}$ & $(10 / 47,2 / 50)$ & $6.49(1.34-31.42)$ & $0.02 p$ & $6.49(1.34-31.42)$ & $0.02 *$ & $18.00(1.59-203.45)$ & $0.02 *$ \\
\hline
\end{tabular}

$\mathrm{Cl}$, confidence interval; OR, odds ratio.

${ }^{a}$ Analyzed with age and sex; ${ }^{b}$ analyzed with age, sex, body mass index, systolic blood pressure, diabetes mellitus, liver cirrhosis, cancer, serum sodium/ creatinine/uric acid/C-reactive protein/serum osmolality/urine osmolality/urine sodium at baseline, cause of hyponatremia, hypertonic saline volume, and infusion mode of hypertonic saline (bolus/continuous/mixed).

$* p<0.05$.

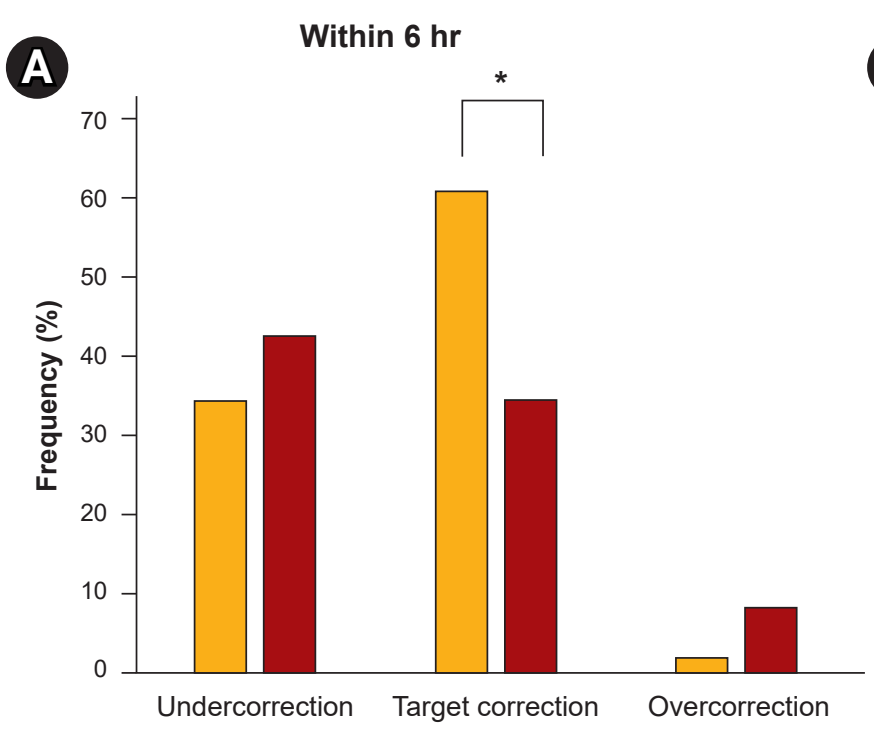

B

Copeptin at baseline

$\square$ Below median

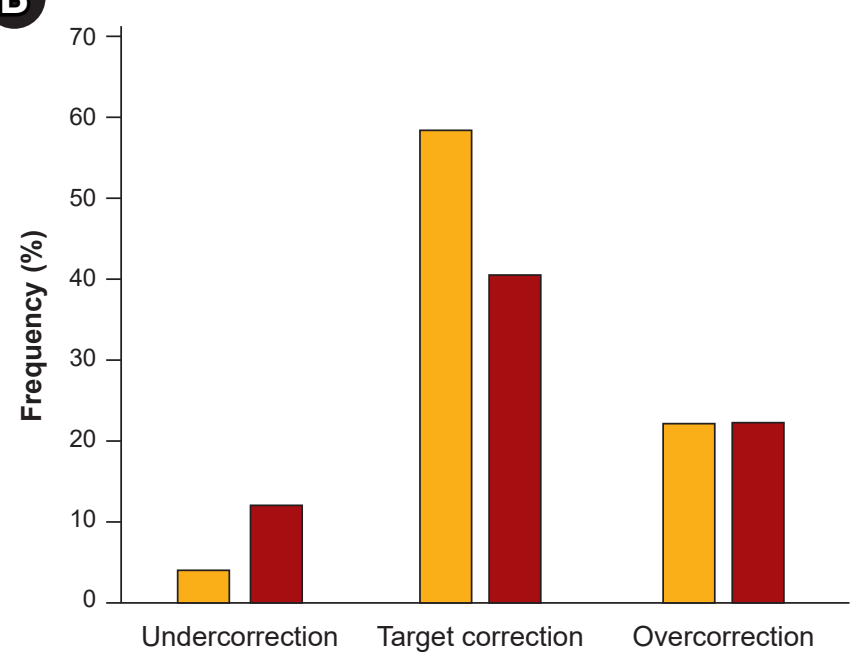

Figure 4. Outcomes within 6 hours (A) and 24 hours (B) stratified by copeptin level at baseline (below/above median).

regression analysis revealed associations between copeptin levels at 24 hours after treatment and outcomes within 24 to 48 hours. Patients with copeptin levels below the median at 24 hours after treatment had an increased risk of overcorrection within 24 to 48 hours (OR, 18.00; 95\% CI, 1.59-203.45; p $=0.02$ ) (Fig. 5). None of the patients developed ODS during the study period.

\section{Discussion}

In this prospective cohort study, copeptin and copeptin-to$\mathrm{UNa}$ ratios showed limited diagnostic utility in the differential diagnosis of hyponatremia. Hyponatremic patients with below-median copeptin levels at baseline achieved a higher target correction rate within $-6 / 24$ hours, while those with below-median copeptin levels 24 hours after treatment expe- 


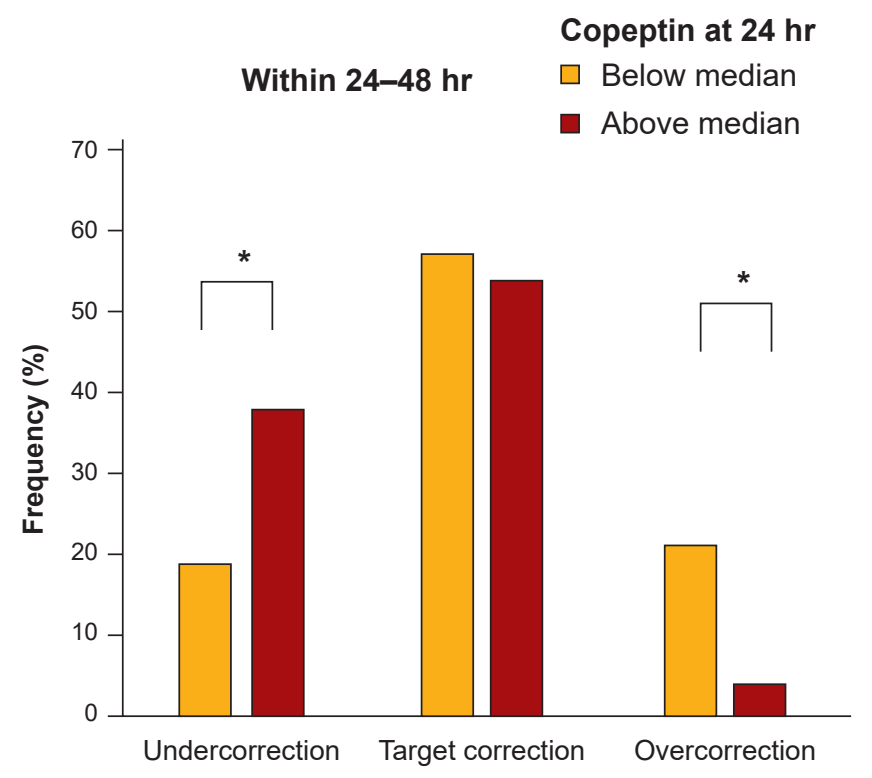

Figure 5. Outcomes within $\mathbf{2 4}$ to $\mathbf{4 8}$ hours stratified by copeptin level at $\mathbf{2 4}$ hours after treatment (below/above median).

rienced a higher overcorrection rate within the next 24 hours.

In this study, the median copeptin level was $16.9 \mathrm{pmol} /$ $\mathrm{L}$, which is similar to the level reported in previous studies when classified according to the cause of hyponatremia $[24,29]$. As shown in Table 4, determinants of copeptin levels were sNa and serum creatinine, in accordance with previous studies [29,30]. High copeptin levels were associated with low sNa and high serum creatinine levels.

We categorized the causes of hyponatremia into five groups and evaluated the diagnostic validity of copeptin for differentiating between the underlying etiologies of hyponatremia. In agreement with a previous study, we observed that while the copeptin level by itself did not have any diagnostic utility, the copeptin-to-UNa ratio allowed for improved differentiation among some subgroups (distinguishing conditions of a secondary AVP surge from a primary AVP release), but not all hyponatremia etiologic subgroups [29]. The copeptin-to-UNa ratio was highest in patients with decreased ECF volume due to non-renal Na loss compared to other etiologies. These observations are similar to those of Nigro et al. [22]. Although copeptin in addition to volume status improved the identification of hypovolemic hyponatremia, copeptin did not seem to have a higher diagnostic value compared with UNa. The copeptin-to-UNa ratio was higher in groups with a low effective arterial blood volume (decreased ECF volume due to non-renal Na loss and the increased ECF group), in other words, secondary AVP release. These two groups had higher serum copeptin levels and lower UNa levels, which resulted in a higher ratio. In agreement with previous reports, copeptin levels widely overlapped among the different etiologies of hyponatremia, and also showed large variability within a single category. One of the possible explanations for this observation is that hyponatremia is not only caused by inappropriate AVP secretion, but also by other factors including comorbidities [17,21], medications, dehydration [23], or stress [31]. Additionally, the correlation between the changes in copeptin levels over the course of 24 hours and the etiologies of hyponatremia were not statistically significant (data not shown).

We also evaluated the efficacy of copeptin as a predictive marker of treatment response. Patients with a baseline serum copeptin level below the median achieved target correction of sNa within 6 hours of treatment. Copeptin and AVP respond to either a decreased ECF volume or increased osmolality to maintain fluid homeostasis [10]. When baseline copeptin levels are low, it could be considered that there is less chance of having previous hyperosmolar or hypovolemic stimuli; sNa may respond better to a hypertonic saline infusion. Conversely, when baseline copeptin levels are high, achieving target correction by hypertonic saline treatment may be more difficult.

Meanwhile, patients with copeptin levels below the median at 24 hours after treatment showed a higher frequency of overcorrection within 24 to 48 hours after treatment. Plasma copeptin is reported to increase rapidly as osmolality changes in response to hypertonic saline infusion in healthy subjects [32]; however, a low copeptin level 24 hours after treatment may indicate a somewhat decreased antidiuretic response during hypertonic saline infusion. A low copeptin level, and thus a low AVP level, at 24 hours after treatment indicates that a greater excretion of free water can occur [33], and the effect of increasing sNa for the same amount of hypertonic saline can be augmented.

It is still unclear why some patients present with low copeptin levels, while others present with high copeptin levels despite receiving the same hypertonic saline treatment, given that most hyponatremic states are characterized by an inappropriate antidiuresis. We present two possible explanations for this finding. First, the atrial natriuretic protein (ANP) may play a role in this complicated response. Previous studies have demonstrated an immediate release of ANP in re- 
sponse to a hypertonic saline infusion, regardless of the etiology of hypernatremia $[34,35]$. Seeing that ANP inhibits not only the secretion of AVP, but also the antidiuretic response itself [36], this may lead to a decrease in AVP levels due to the negative feedback of ANP on AVP. This could explain the low copeptin levels in some of the patients included in our study. Recently, Nigro et al. [37] reported an association between midregional proANP and the differential diagnosis of hyponatremia; the midregional proANP levels were prominently higher in the hypervolemic hyponatremia group at baseline. It may not respond normally to hypertonic saline in conditions with chronic hypervolemic hyponatremia, such as heart failure or liver cirrhosis. If low copeptin levels are observed 24 hours after treatment, it is necessary to reevaluate the change in volume status or other comorbidities before continuing with the hypertonic saline infusion.

The second possible explanation for the low copeptin level at 24 hours after treatment is the pathological heterogeneity of SIAD. In our study, $39 \%$ of the patients were diagnosed with SIAD. Fenske et al. [8] suggested a copeptin-based classification of SIAD subtypes, and stated that there were five subtypes according to the changes in copeptin levels during a hypertonic saline treatment. One-fourth of the SIAD patients presented with elevated copeptin levels, while the remaining patients were in the normal or below normal range despite hypertonic saline infusion [8]. Due to the varying degree of osmoregulatory defects, it is necessary to pay attention to the correction rate of sNa during hypertonic saline treatment in SIAD, especially when the copeptin levels after 24 hours of treatment are low. We divided our population into five groups (designated A-E) according to copeptin levels measured at baseline and at 24 hours as in a previous study [8]. We then analyzed the association between $\mathrm{A}$ and E grouping and outcomes within 24 hours. Among all patients, $2.2 \%$, $9.7 \%, 40.9 \%, 1.1 \%$, and $46.2 \%$ of patients were in groups A to $\mathrm{E}$. However, this classification was not statistically significant in predicting outcomes (target, under-, and overcorrection rates of sNa) within 24 hours (data not shown). It is presumed that the number of patients was too small to prove the relevance of these classifications and outcomes.

In hyponatremic patients, serum copeptin at baseline predicted a target correction within 6 hours of hypertonic saline treatment, thereby serving as an efficacy index. Furthermore, copeptin at 24 hours after treatment predicted an overcorrection rate within 24 to 48 hours posttreatment, thus serving as a safety index. The clinical application of our findings lies in the suggestion that for patients with low copeptin levels, a bolus therapy of hypertonic saline is more useful than a continuous infusion because bolus therapy limits the risk of overcorrection, which is commonly associated with continuous infusion $[1,38]$.

In acute illness, the secretion of AVP and copeptin may be driven by nonosmotic stimuli [31]. In this study, patients with anemia or acute inflammation/infection were also included. Therefore, we performed sensitivity analysis for the association between copeptin and outcomes, excluding patients with anemia (hemoglobin $\leq 10 \mathrm{~g} / \mathrm{dL}, \mathrm{n}=16$ ) or acute inflammation (C-reactive protein $\geq 50 \mathrm{mg} / \mathrm{L}, \mathrm{n}=23$ ). In patients with hemoglobin levels above $10 \mathrm{~g} / \mathrm{dL}(\mathrm{n}=84)$, similar results were obtained. However, in patients with C-reactive protein levels below $50 \mathrm{mg} / \mathrm{dL}(\mathrm{n}=77)$, the association of copeptin at baseline with the target correction within 24 hours, and the association of copeptin at 24 hours after treatment with overcorrection within 24 to 48 hours, was statistically significant (Supplementary Table 3, available online).

This study has several limitations. First, the sample size of 100 patients with hyponatremia is somewhat small. Second, while we evaluated the correction rate or the magnitude of sNa in the treatment of hyponatremia, no patient developed ODS, which is the true outcome of interest despite overcorrection; however, several studies have reported that overcorrection may be one of the main causes of ODS $[5,39,40]$. Overcorrection may be a good laboratory outcome and may be resolved by relowering the infused amount in clinical practice [28]. Third, copeptin is not commercially available at present, similar to other biomarker studies. We acknowledge that it is important to improve the prognosis by repeatedly measuring sNa to achieve the target correction rate in the treatment of hyponatremia. We hypothesized that copeptin might be useful as an additional factor to sNa itself in treating hyponatremic patients by suggesting the methods of hypertonic saline administration. Fourth, 38 participants from the prospective cohort did not receive hypertonic saline with the formalized protocol as bolus therapy or continuous infusion. However, there was no difference in distribution between bolus and continuous infusion in all patients, and we tried to reduce potential confounding by adjusting hypertonic saline volume for 48 hours in multivariable analysis. Fifth, we did not include healthy controls. Finally, we did not routinely measure serum or urine osmolality after 
hypertonic saline infusion. Thus, we could not examine the association between copeptin levels and osmolality to predict treatment response.

Nonetheless, this is the first study to demonstrate the usefulness of copeptin in predicting the efficacy and safety of hypertonic saline treatment in patients with hyponatremia. We suggest that measuring copeptin in addition to sNa itself may help physicians achieve proper correction of hyponatremia. Further studies involving a large number of patients and a formalized infusion protocol for hypertonic saline are required.

\section{Conflicts of interest}

All authors have no conflicts of interest to declare.

\section{Funding}

The research was funded by Hallym University Research Fund (HURF-2018-29), the Korean Association of Internal Medicine (2018-180), the Korean Society of Nephrology, and National Research foundation of Korea (2016R1C1B1015687).

\section{Authors' contributions}

Conceptualization: SK, SHB

Investigation: All authors

Data curation, Formal analysis: SG, SK, SHB

Administrative, Technical, or Material support: HES, JYR, HY, SRC, JWS, YHJ, JRK

Funding acquisition: SHB

Writing-original draft: SG, SK, SHB

Writing-review \& editing: All authors

\section{ORCID}

Suryeong Go, https://orcid.org/0000-0001-8118-7994 Sejoong Kim, https://orcid.org/0000-0002-7238-9962 Hyung-Eun Son, https://orcid.org/0000-0002-8719-3823

Ji-Young Ryu, https://orcid.org/0000-0003-4134-1007 Huijin Yang, https://orcid.org/0000-0001-5885-1519 Sun Ryoung Choi, https://orcid.org/0000-0002-9668-3349 Jang-Won Seo, https://orcid.org/0000-0002-3495-5388 You Hwan Jo, https://orcid.org/0000-0002-9507-7603 Ja-Ryong Koo, https://orcid.org/0000-0003-4245-2569 Seon Ha Baek, https://orcid.org/0000-0002-4751-9817

\section{References}

1. Spasovski G, Vanholder R, Allolio B, et al. Clinical practice guideline on diagnosis and treatment of hyponatraemia. Eur J Endocrinol 2014;170:G1-G47.

2. Adrogué HJ, Madias NE. Hyponatremia. N Engl J Med 2000; 342:1581-1589.

3. Gross P, Reimann D, Neidel J, et al. The treatment of severe hyponatremia. Kidney Int Suppl 1998;64:S6-S11.

4. Chawla A, Sterns RH, Nigwekar SU, Cappuccio JD. Mortality and serum sodium: do patients die from or with hyponatremia? Clin J Am Soc Nephrol 2011;6:960-965.

5. Sterns RH, Riggs JE, Schochet SS Jr. Osmotic demyelination syndrome following correction of hyponatremia. N Engl J Med 1986;314:1535-1542.

6. Verbalis JG, Martinez AJ. Neurological and neuropathological sequelae of correction of chronic hyponatremia. Kidney Int 1991;39:1274-1282.

7. Verbalis JG, Goldsmith SR, Greenberg A, et al. Diagnosis, evaluation, and treatment of hyponatremia: expert panel recommendations. Am J Med 2013;126(10 Suppl 1):S1-S42.

8. Fenske W, Sandner B, Christ-Crain M. A copeptin-based classification of the osmoregulatory defects in the syndrome of inappropriate antidiuresis. Best Pract Res Clin Endocrinol Metab 2016;30:219-233.

9. Refardt J, Winzeler B, Christ-Crain M. Copeptin and its role in the diagnosis of diabetes insipidus and the syndrome of inappropriate antidiuresis. Clin Endocrinol (Oxf) 2019;91:22-32.

10. Christ-Crain M, Fenske W. Copeptin in the diagnosis of vasopressin-dependent disorders of fluid homeostasis. Nat Rev Endocrinol 2016;12:168-176.

11. Struck J, Morgenthaler NG, Bergmann A. Copeptin, a stable peptide derived from the vasopressin precursor, is elevated in serum of sepsis patients. Peptides 2005;26:2500-2504.

12. Land H, Schütz G, Schmale H, Richter D. Nucleotide sequence of cloned cDNA encoding bovine arginine vasopressin-neurophysin II precursor. Nature 1982;295:299-303.

13. Morgenthaler NG, Struck J, Alonso C, Bergmann A. Assay for the measurement of copeptin, a stable peptide derived from the precursor of vasopressin. Clin Chem 2006;52:112-119.

14. Fenske W, Quinkler M, Lorenz D, et al. Copeptin in the differential diagnosis of the polydipsia-polyuria syndrome: revisiting the direct and indirect water deprivation tests. J Clin Endocrinol Metab 2011;96:1506-1515.

15. Fenske W, Refardt J, Chifu I, et al. A copeptin-based approach in 
the diagnosis of diabetes insipidus. N Engl J Med 2018;379:428439.

16. Giannitsis E, Kehayova T, Vafaie M, Katus HA. Combined testing of high-sensitivity troponin $\mathrm{T}$ and copeptin on presentation at prespecified cutoffs improves rapid rule-out of non-ST-segment elevation myocardial infarction. Clin Chem 2011;57:1452-1455.

17. Maisel A, Mueller C, Neath SX, et al. Copeptin helps in the early detection of patients with acute myocardial infarction: primary results of the CHOPIN trial (Copeptin Helps in the early detection Of Patients with acute myocardial INfarction). J Am Coll Cardiol 2013;62:150-160.

18. Jochberger S, Dörler J, Luckner G, et al. The vasopressin and copeptin response to infection, severe sepsis, and septic shock. Crit Care Med 2009;37:476-482.

19. Meijer E, Bakker SJ, van der Jagt EJ, et al. Copeptin, a surrogate marker of vasopressin, is associated with disease severity in autosomal dominant polycystic kidney disease. Clin J Am Soc Nephrol 2011;6:361-368.

20. Voors AA, von Haehling S, Anker SD, et al. C-terminal provasopressin (copeptin) is a strong prognostic marker in patients with heart failure after an acute myocardial infarction: results from the OPTIMAAL study. Eur Heart J 2009;30:1187-1194.

21. Moreno JP, Grandclement E, Monnet E, et al. Plasma copeptin, a possible prognostic marker in cirrhosis. Liver Int 2013;33:843-851.

22. Nigro N, Winzeler B, Suter-Widmer I, et al. Evaluation of copeptin and commonly used laboratory parameters for the differential diagnosis of profound hyponatraemia in hospitalized patients: 'The Co-MED Study'. Clin Endocrinol (Oxf) 2017;86:456-462.

23. Boursier G, Alméras M, Buthiau D, et al. CT-pro-AVP as a tool for assessment of intravascular volume depletion in severe hyponatremia. Clin Biochem 2015;48:640-645.

24. Nigro N, Müller B, Morgenthaler N, et al. The use of copeptin, the stable peptide of the vasopressin precursor, in the differential diagnosis of sodium imbalance in patients with acute diseases. Swiss Med Wkly 2011;141:w13270.

25. Baek SH, Jo YH, Ahn S, et al. Risk of overcorrection in rapid intermittent bolus vs slow continuous infusion therapies of hypertonic saline for patients with symptomatic hyponatremia: the SALSA randomized clinical trial. JAMA Intern Med 2021;181:81-92.

26. Lee A, Jo YH, Kim K, et al. Efficacy and safety of rapid intermittent correction compared with slow continuous correction with hypertonic saline in patients with moderately severe or severe symptomatic hyponatremia: study protocol for a randomized controlled trial (SALSA trial). Trials 2017;18:147.

27. Oh SJ, Ihm CG, Lee TW, et al. Syndrome of inappropriate an- tidiuretic hormone secretion associated with seronegative neuromyelitis optica spectrum disorder. Kidney Res Clin Pract 2017;36:100-104.

28. Woodfine JD, Sood MM, MacMillan TE, Cavalcanti RB, van Walraven C. Derivation and validation of a novel risk score to predict overcorrection of severe hyponatremia: the Severe Hyponatremia Overcorrection Risk (SHOR) Score. Clin J Am Soc Nephrol 2019;14:975-982.

29. Fenske W, Störk S, Blechschmidt A, Maier SG, Morgenthaler NG, Allolio B. Copeptin in the differential diagnosis of hyponatremia. J Clin Endocrinol Metab 2009;94:123-129.

30. Meijer E, Bakker SJ, Halbesma N, de Jong PE, Struck J, Gansevoort RT. Copeptin, a surrogate marker of vasopressin, is associated with microalbuminuria in a large population cohort. Kidney Int 2010;77:29-36.

31. Katan M, Christ-Crain M. The stress hormone copeptin: a new prognostic biomarker in acute illness. Swiss Med Wkly 2010;140: w13101.

32. Szinnai G, Morgenthaler NG, Berneis K, et al. Changes in plasma copeptin, the c-terminal portion of arginine vasopressin during water deprivation and excess in healthy subjects. J Clin Endocrinol Metab 2007;92:3973-3978.

33. Bankir L, Bichet DG, Morgenthaler NG. Vasopressin: physiology, assessment and osmosensation. J Intern Med 2017;282:284-297.

34. Arjamaa O, Karlqvist K, Kanervo A, Vainionpää V, Vuolteenaho O, Leppäluoto J. Plasma ANP during hypertonic $\mathrm{NaCl}$ infusion in man. Acta Physiol Scand 1992;144:113-119.

35. Clark BA, Sclater A, Epstein FH, Elahi D. Effect of glucose, insulin, and hypertonicity on atrial natriuretic peptide levels in man. Metabolism 1993;42:224-228.

36. Inoue T, Nonoguchi H, Tomita K. Physiological effects of vasopressin and atrial natriuretic peptide in the collecting duct. Cardiovasc Res 2001;51:470-480.

37. Nigro N, Winzeler B, Suter-Widmer I, et al. Mid-regional pro-atrial natriuretic peptide and the assessment of volaemic status and differential diagnosis of profound hyponatraemia. J Intern Med 2015;278:29-37.

38. Hoorn EJ, Zietse R. Diagnosis and treatment of hyponatremia: compilation of the guidelines. J Am Soc Nephrol 2017;28:13401349.

39. Sterns RH, Cappuccio JD, Silver SM, Cohen EP. Neurologic sequelae after treatment of severe hyponatremia: a multicenter perspective. J Am Soc Nephrol 1994;4:1522-1530.

40. Berl T. Treating hyponatremia: damned if we do and damned if we don't. Kidney Int 1990;37:1006-1018. 